\title{
Atividade larvicida de Anacardium occidentale como alternativa ao controle de Aedes aegypti e sua toxicidade em Rattus norvegicus
}

GUISSONI, A.C.P.'; SILVA, I.G.'; GERIS, R. ${ }^{2}$; CUNHA, L.C. '; SILVA, H.H.G. ${ }^{\text {* }}$

'Universidade Federal de Goiás, ${ }^{\text {a }}$. Avenida s/n, Setor Universitário, CEP 74605-050, Caixa Postal 131, Goiânia, GO. *garciaheloisa@yahoo.com.br, ${ }^{2}$ Universidade Federal da Bahia, Rua Barão de Geremoabo, 147, Campus de Ondina, CEP 40170-115, Salvador, BA.

RESUMO: A busca por substitutos para os inseticidas sintéticos tem estimulado muitos trabalhos científicos contemplando inclusive a utilização de óleos, extratos, ou constituintes ativos provenientes de plantas. Esta procura pode ser justificada pelo potencial inseticida associado à fácil degradação de seus constituintes, menor toxicidade ao homem e uma alternativa mais segura para o meio ambiente. Após a coleta e aquecimento dos frutos de Anacardium occidentale (Anacardiaceae) a $40^{\circ} \mathrm{C}$, obteve-se um líquido da castanha de caju (LCC) que, depois de testado quanto à sua atividade larvicida, foi fracionado em coluna de sílica gel dando origem a oito frações, as quais foram codificadas como $\mathrm{AO}_{1}$ a $\mathrm{AO}_{8}$ e submetidas a ensaios larvicidas. Avaliou-se também sua toxicidade oral aguda em Rattus norvegicus. O LCC e as frações $\mathrm{AO}_{2} \mathrm{e}$ $\mathrm{AO}_{3}$ apresentaram atividade larvicida para Aedes aegypti. As concentrações letais, $\mathrm{CL}_{50}$ e $\mathrm{CL}_{90}$ do $\mathrm{LCC}$ foram, respectivamente, de 6,55 e 10,98 ppm. Para $\mathrm{AO}_{2}$ e $\mathrm{AO}_{3}$, as $\mathrm{CL}_{50}$ e $\mathrm{CL}_{90}$ foram de 3,18 e 7,80 ppm, e de 3,57 e 10,47 ppm, respectivamente. Não foi observada nenhuma toxicidade do LCC e das frações para $R$. norvegicus. O LCC e as frações foram administrados por via oral na dose de $2000 \mathrm{mg} / \mathrm{kg}$. Esses produtos apresentaram potencial larvicida sobre Ae. aegypti e nenhum sinal de toxicidade foi evidenciado nos parâmetros analisados.

Palavras-chave: Anacardium occidentale, Aedes aegypti, larvicida, toxicidade aguda.

ABSTRACT: Larvicidal activity of Anacardium occidentale as an alternative to control Aedes aegypti and its toxicity in Rattus norvegicus. The search for substitutes for synthetic pesticides has been the subject of many scientific publications, including considering the use of oils, extracts and active constituents from plants. This demand can be justified by the insecticide potential associated with an easy degradation of its constituents, lower toxicity to humans and, consequently, a safer alternative for the environment. After collecting and heating the Anacardium occidentale (Anacardiaceae) fruits at $40^{\circ} \mathrm{C}$, they were tested to confirm their larvicidal activity. Then, it was fractionated in a silica gel column. The fractionation resulted in eight fractions, which were coded as $\mathrm{AO}_{1}$ to $\mathrm{AO}_{8}$. In this paper, the cashew nut shell liquid (CNSL) and its fractions were evaluated as to their biological activity in the third instar larvae of Aedes aegypti. The acute oral toxicity in Rattus norvegicus also was evaluated. CNSL and the $\mathrm{AO}_{2}$ and $\mathrm{AO}_{3}$ fractions presented larvicidal activity. The lethal concentrations, $\mathrm{LC}_{50}$ and $\mathrm{LC}_{90}$, of CNSL were, respectively, 6.55 and $10.98 \mathrm{ppm}$. The active fractions, $\mathrm{AO}_{2}$ and $\mathrm{AO}_{3}$, presented $\mathrm{LC}_{50}$ and $\mathrm{LC}_{90}$ of 3.18 and of $7.80 \mathrm{ppm}$, and 3.57 and $10.47 \mathrm{ppm}$, respectively. The LCC and the fractions were orally administered at a dose of $2000 \mathrm{mg} / \mathrm{kg}$. These products showed larvicidal potential against $A e$. aegypti and no sign of toxicity was evident in the parameters analyzed.

Keywords: Anacardium occidentale, Aedes aegypti, larvicidal, acute toxicity.

\section{INTRODUÇÃO}

A dengue é uma doença causada por um arbovírus da família Flaviviridae e gênero Flavivirus, que apresenta quatro sorotipos infectantes (Figueiredo, 2000; WHO, 2009). O vetor mais importante em toda faixa intertropical do globo terrestre é o Aedes aegypti (Tauil, 2001; Braga \& Valle, 2007; WHO, 2009). Como ainda não estão disponíveis medicamentos antivirais para tratamento,

Recebido para publicação em 18/04/2012

Aceito para publicação em 21/11/2012

Rev. Bras. PI. Med., Campinas, v.15, n.3, p.363-367, 2013. 
nem uma vacina eficaz para uso humano, o controle da dengue se baseia, principalmente, em aplicações de inseticidas (WHO, 2009). O uso continuado de inseticida fez surgir populações resistentes de Ae. aegypti (Macoris et al., 2003), dificultando o êxito dos programas de controle.

Compostos derivados de plantas têm sido apresentados como alternativas ao controle de vetores, não só como novos agentes inseticidas, mas também por serem ambientalmente mais seguros (Mendonça et al. 2005; Omena et al. 2007; Garcez et al., 2009; Mukhopadhyay et al. 2010; Santos et al. 2010). No entanto, para serem registrados como inseticidas, os compostos devem ser avaliados em relação aos seus efeitos toxicológicos, conforme padronização internacional (Zucker, 1985; OECD, 2004).

Anacardium occidentale (Anacardiaceae) é uma planta conhecida popularmente por cajueiro; pertence à família Anacardiaceae e apresenta grande importância econômica no Brasil. Seu pedúnculo é confundido com o fruto, sendo muito apreciado pela suculência. No entanto, o fruto é a castanha e dela se retira um líquido escuro, quase preto, denominado por Mazzetto et al. (2009) de líquido da castanha do caju (LCC) ou cashew nut shell liquid (CNSL). Para a obtenção do LCC, empregamse diferentes processos como a extração a frio, extração por solventes (Correia et al., 2006) ou processo térmico-mecânico, onde o próprio LCC quente é utilizado para aquecer as castanhas a $190^{\circ} \mathrm{C}$. Quando submetido a altas temperaturas o ácido anacárdico sofre reação de descarboxilação convertendo-se a cardanol, produzindo o denominado LCC técnico (Lopes, 2005; Rios, 2008).

Apresentam-se, neste trabalho, o estudo da atividade larvicida do LCC e de frações de A. occidentale sobre o Ae. aegypti, e também a toxicidade oral aguda em Rattus norvegicus, visando encontrar alternativas naturais, de baixa toxicidade para vertebrados, sem impacto ambiental, que possam ser utilizadas no controle desse mosquito.

\section{MÉTODOS E MÉTODOS}

\section{Material botânico}

Frutos de $A$. occidentale $\mathrm{L}$. foram colhidos no setor Pedro Ludovico, em Goiânia, GO, Brasil, no mês de outubro de 2008. Uma exsicata foi autenticada pelo Professor José Ângelo Rizzo e depositada no Herbário da Unidade de Conservação, sob n 43.180, no Departamento de Botânica da Universidade Federal de Goiás (UFG).

\section{Obtenção e Fracionamento do Líquido da Castanha de A. occidentale}

No Laboratório de Bioatividade de Plantas do Instituto de Patologia Tropical e Saúde Pública (IPTSP) da UFG, dois kg de frutos foram colocados em estufa de ventilação forçada, a $40^{\circ} \mathrm{C}$, por 7 dias. Com esse procedimento foram obtidos $600 \mathrm{~g}$ de um líquido de coloração marrom e de odor forte, denominado líquido da castanha do caju (LCC). Inicialmente, $50 \mathrm{~g}$ do líquido obtido de $A$. occidentale foram submetidos a uma filtração a vácuo, em um funil de placa sinterizada, com diâmetro interno de $9 \mathrm{~cm}$ e uma altura de gel de sílica (70-230 mesh) de $15 \mathrm{~cm}$. As frações foram eluídas, seguindo a técnica de gradiente com n-hexano, acetato de etila e metanol, totalizando oito frações. Estas foram denominadas, em ordem crescente, $\mathrm{AO}_{1}$ a $\mathrm{AO}_{8} \mathrm{e}$ submetidas aos ensaios larvicidas.

\section{Bioensaios em Laboratório}

Os bioensaios foram realizados com larvas de $3^{\circ}$ estádio de Ae. aegypti, por serem as mais tolerantes em relação aos demais estádios (Silva et al., 2003). O líquido e/ou as frações foram primeiramente pesados e pré-solubilizados em dimetilsulfóxido (DMSO). A quantidade de solvente utilizada para o preparo da solução foi previamente determinada por ensaios de tolerância sem mortalidade das larvas, e foi de $0,4 \%$. Para cada uma das amostras a serem testadas preparou-se uma solução-mãe, acrescentando-se água, num volume suficiente para obter a concentração de $100 p p m$ para o líquido e as frações. A partir destas soluções uma série de diluições foi preparada a fim de se obterem concentrações menores de 80, 60, $40,20,10,5$ e $2,5 p p m\left(C_{1} \cdot V_{1}=C_{2} \cdot V_{2}\right)$. Os bioensaios foram realizados em copos com capacidade para 30 $\mathrm{mL}$. Nestes, foram colocados $25 \mathrm{~mL}$ de cada uma das soluções e em seguida 20 larvas de $3^{\circ}$ estádio. Todos os bioensaios foram realizados em triplicata com duas repetições.

As leituras da mortalidade foram feitas após $24 \mathrm{~h}$ de exposição das larvas às soluções. Todos os experimentos foram acompanhados de uma série controle, contendo o mesmo número de larvas e o mesmo volume de DMSO e água destilada.

A avaliação de toxicidade aguda foi realizada em $R$. norvegicus (linhagem Wistar) de ambos os sexos, oriundos do biotério central da UFG. Os animais eram adultos jovens, com 8 a 12 meses de idade e o peso de cada um não excedeu a $20 \%$ da média do grupo, que foi de $300 \mathrm{~g}$ para machos e $280 \mathrm{~g}$ para fêmeas.

Os animais foram mantidos no biotério do Núcleo de Estudos e Pesquisas TóxicoFarmacológicas (NEPET) da Faculdade de Farmácia da UFG. Foram alimentados com ração balanceada 
e água filtrada. O protocolo experimental foi aprovado pelo Comitê de Ética em Pesquisa Animal da Universidade Federal de Goiás (Protocolo $n^{\circ}$ 292/10), seguindo as diretrizes da OECD (2004). Para realização do experimento, dois grupos foram formados, com três machos e três fêmeas cada um. Por se tratar de um extrato de origem vegetal com finalidade inseticida e pela inexistência de casos de intoxicação, os experimentos foram realizados na dose única de $2000 \mathrm{mg} / \mathrm{kg}$ de LCC, considerada limite superior para vegetais. Administrou-se $1 \mathrm{~mL}$ da solução para cada $100 \mathrm{~g}$ de peso corporal, por via oral, a cada animal. Simultaneamente, o grupo controle foi realizado com o mesmo número de animais, e solução de água e DMSO $(0,4 \%)$. As observações foram feitas, seguindo o screening hipocrático (atividade geral, frênito vocal, irritabilidade, reposta ao toque, resposta ao aperto da cauda, contorção, força para agarrar, tremores, convulsões, salivação, lacrimejamento, micção, defecação, piloereção, morte) por um período de 14 dias. No $15^{\circ}$ dia, os ratos foram pesados, anestesiados, pela via intraperitoneal, com cetamina a $5 \%$ e xilazina a $10 \%$, depois, eutanasiados e os órgãos foram analisados macroscopicamente.

As observações do screening hipocrático foram registradas em protocolo impresso com a lista dos sinais investigados. As intensidades dos eventos foram tabuladas de zero a quatro, correspondendo, respectivamente a ausente (0), raro (1), pouco (2), moderado (3) e intenso (4), até o décimo quarto dia (Malone \& Robichaud, 1962).

Os dados obtidos da mortalidade $x$ concentração (ppm) foram analisados pelo programa SAEG (Sistema de Análises Estatísticas), Versão 9.1, em gráfico de Probit, para determinar as concentrações letais $\left(\mathrm{CL}_{50 \text { e } 90}\right)$ e os respectivos intervalos de confiança (IC). A toxicidade aguda foi analisada pelo teste de Student $(t)$, ao nível de $5 \%$, através do programa Signa Plot.

\section{RESULTADOS}

Utilizando-se como método de obtenção o aquecimento das castanhas a $40^{\circ} \mathrm{C}$, obteve-se, neste trabalho, um rendimento de $30 \%$ de LCC. As concentrações letais do LCC sobre larvas de $3^{\circ}$ estádio de $A e$. aegypti foram de 6,55 e 10,98 ppm, respectivamente, para $\mathrm{CL}_{50}$ e $\mathrm{CL}_{90}$. Após a separação cromatográfica foi possível obter concentrações letais mais baixas com as frações $\mathrm{AO}_{2}$ e $\mathrm{AO}_{3}$ (Tabela 1).

Do fracionamento do LCC obtiveram-se 8 frações, mas somente $\mathrm{AO}_{2}$ e $\mathrm{AO}_{3}$ apresentaram atividade larvicida e o rendimento foi de $43 \%$ e $15,7 \%$, respectivamente.

A toxicidade oral aguda LCC avaliada em
TABELA 1: Atividade larvicida, em laboratório, do líquido e das frações de Anacardium occidentale sobre larvas de $3^{\circ}$ estádio de Aedes aegypti.

\begin{tabular}{lll}
\hline Frações & CL $_{50}$ (IC 95\%) ppm & CL $_{90}$ (IC 95\%) ppm \\
\hline LCC & $6,55(6,09-6,98)$ & $10,98(10,04-12,44)$ \\
$\mathrm{AO}_{2}$ & $3,18(2,70-3,64)$ & $7,80(6,77-9,31)$ \\
$\mathrm{AO}_{3}$ & $3,57(2,99-4,13)$ & $10,47(8,71-13,49)$ \\
\hline
\end{tabular}

LCC - líquido da castanha do caju; CL - Concentração Letal; IC - Intervalo de Confiança a 95\%

ppm - partes por milhão; AO - Anacardium occidentale

$R$. norvegicus pelo screening hipocrático, na dose administrada de $2000 \mathrm{mg} / \mathrm{kg}$, não causou nenhum sinal significativo de intoxicação aos animais, quando comparado ao grupo controle, pelo teste $t$, ao nível de $5 \%$.

\section{DISCUSSÃO}

Há no mundo uma crescente preocupação relacionada à preservação do meio ambiente. Liderado pelas nações mais desenvolvidas, um movimento de nova consciência ambiental se espalha, gerando organizações, reuniões e discussões entre países para promover mudanças de condutas para um novo modelo de vida. Com isso, tem aumentado progressivamente estudos com plantas com diferentes finalidades. Dentre essas, a atividade inseticida das plantas e a sua baixa toxicidade para mamíferos tem sido motivos de várias investigações. Existem outros fatores que, associados aos motivos anteriores, criam expectativas maiores, com os subprodutos da indústria alimentícia, como é o caso do líquido da castanha do caju (LCC), cujo potencial inseticida foi avaliado nesse trabalho.

Segundo Rios (2008), o LCC é uma das maiores fontes de lipídios fenólicos de origem natural, tendo como componentes majoritários o ácido anacárdico, cardanol, cardol e 2-metilcardol. Quando submetido a temperaturas em torno de $180^{\circ} \mathrm{C}$, o ácido anacárdico converte-se a cardanol, produzindo o chamado LCC técnico.

A atividade larvicida do LCC técnico, obtido com o aquecimento das castanhas a $190^{\circ} \mathrm{C}$, foi avaliada por Lomonaco et al. (2009) sobre Ae. aegypti, e a $\mathrm{CL}_{50}$ encontrada foi de $51 \mathrm{ppm}$, sendo cerca de 8 vezes maior do que a encontrada neste trabalho. Se o aquecimento converte o ácido anarcárdico a cardanol e diminui a atividade, podemos supor que este ácido seja o responsável por uma maior atividade larvicida. O LCC obtido no presente estudo, com aquecimento a $40^{\circ} \mathrm{C}$, provavelmente tenha maior concentração de ácido anacárdico, responsável pela maior atividade. 
O fracionamento de extratos vegetais, guiado por bioensaios, foi utilizado na tentativa de se potencializar a atividade biológica e, posteriormente, conseguir o isolamento do princípio ativo (Silva et al., 2007; Geris et al., 2008). Porém, isso nem sempre acontece, pois alguns trabalhos mostraram que as substâncias puras isoladas eram menos ativas que suas frações de origem (Simas et al., 2004; Silva et al., 2007). Isto sugere um efeito sinérgico de compostos presentes na fração. Neste trabalho, o fracionamento originou uma fração mais ativa que o LCC inicial.

Os resultados da atividade larvicida do LCC e das frações, obtidos neste trabalho, foram significativos para o controle de Ae. aegypti, quando comparados a outros trabalhos pertinentes da literatura, para a mesma espécie de mosquito (Mendonça et al., 2005; Omena et al., 2007; Farias et al., 2009; Mukhopadhyay et al., 2010; Tripatiiy et al., 2011)

As concentrações letais obtidas com o LCC foram menores do que as encontradas por Silva et al.(2007), com o óleo resina de Copaifera reticulata, de 8,6 e 59,4ppm, para as $\mathrm{CL}_{50}$ e $\mathrm{CL}_{90}$, respectivamente.

Esses resultados favoráveis estimulam a continuidade do estudo, visando o isolamento do princípio ativo e, principalmente, formas que viabilizem o seu uso prático para o controle de $A e$. aegypti.

A estimativa da dose letal mediana oral para causar a morte de $50 \%$ dos animais $\left(L_{50}\right)$ foi maior que $2000 \mathrm{mg} / \mathrm{kg}$. Segundo as diretrizes da OECD (2004), este valor permite classificar o LCC, na classe 5, ou seja, substância de muito baixa toxicidade. O LCC e as frações $\mathrm{AO}_{2}$ e $\mathrm{AO}_{3}$ apresentaram atividade larvicida para $A$ e. aegypti com potencial a ser candidato nas ações de controle desse vetor. O uso do LCC é interessante pela possibilidade de aproveitamento de subprodutos da indústria da castanha, ou, ainda, pela conveniência de ser um recurso renovável e não apresentar nenhum sinal de toxicidade dentro dos parâmetros analisados neste trabalho.

\section{AGRADECIMENTOS}

Ao Professor Ângelo Rizzo do Departamento de Botânica da UFG, pela identificação do material botânico e a FAPEG pelo apoio financeiro.

\section{REFERÊNCIA}

BRAGA, I.A.; VALLE, D. Aedes aegypti: Histórico do Controle no Brasil. Epidemiologia e Serviços de Saúde, v.16, n.2, p.113-18, 2007.

CORREIA, S.J. et al. Metabólitos secundários de espécies de Anacardiaceae. Química Nova, v.29, n.6, p.1287-
300, 2006.

FARIAS, D.F. et al. Insecticidal action of sodium anacardate from brazilian cashew nut Shell liquid against Aedes aegypti. Journal of the American Mosquito Control Association, v.25, n.3, p.386-89, 2009.

FIGUEIREDO, L.T.M. The Brazilian flaviviruses. Microbes and Infection, v.2, n.13, p.1643-49, 2000.

GARCEZ, W.S. et al. Larvicidal activity against Aedes aegypti of some plants native to the West-Central region of Brazil. Bioresource Technology, v.100, n.24, p.6647-50, 2009.

GERIS, R. et al. Diterpenoids from Copaifera reticulata Ducke with larvicidal activity against Aedes aegypti (Diptera: Culicidae). Revista do Instituto de Medicina Tropical de São Paulo, v.50, n.1, p.25-8, 2008.

LOMONACO, D. et al. Study of technical CNSL and its main components as new green larvicides. Green Chemistry, v.11, n.1, p.31-3, 2009.

LOPES, A.A.S. Síntese de um aditivo tiofosforado a partir do líquido da casca da castanha de caju (Anacardium occidentale Lin). 2005. 125p. Dissertação (Mestrado em Engenharia Química) Universidade Federal do Ceará, Fortaleza.

MACORIS, M.L. et al. Resistance of Aedes aegypti from the state of São Paulo, Brazil, to organophosphates insecticides. Memórias do Instituto Oswaldo Cruz, v.98, n.5, p.703-8, 2003.

MALONE, M.H.; ROBICHAUD, R.C. A hipocratic screen for pure or crud drug materials. Loydia, 1962. p.32032.

MAZZETTO, S.L. et al. Óleo da Castanha de caju: Oportunidades e Desafios no Contexto do desenvolvimento e Sustentabilidade industrial. Química Nova, v.32, n.3, p.732-41, 2009.

MENDONÇA, F.A.C. et al. Activities of some Brazilian plants against larvae of the Aedes aegypti. Fitoterapia, v.76, n.7, p.629-36, 2005.

MUKHOPADHYAY, A.K. et al. Larvicidal properties of cashew nut shell liquid (Anacardium occidentale L.) on immature stages of two mosquito species. Journal Vector Borne, v.47, n.12, p.257-60, 2010.

OECD-ORGANIZATION FOR ECONOMIC COOPERATION AND DEVELOPMENT. Guidelines for testing of chemicals (OECD-423). Paris: OECD/ OCDE, 2004. 275p.

OMENA, M.C. et al. Larvicidal activities against Aedes aegypti of some Brazilian plants. Bioresource Technology, v.98, n.13, p.2549-56, 2007.

RIOS, M.A. Síntese e aplicabilidade de antioxidantes derivados do cardanol hidrogenado. 2008. Tese (Doutorado - área de concentração Química Inorgânica) - Universidade Federal do Ceará, Fortaleza.

SANTOS, E. et al. Oviposition activity of Aedes aegypti L. (Diptera: Culicidae) in response to different organic infusions. Neotropical Entomology, v.39, n.2, p.299302, 2010.

SILVA, H.H.G. et al. Larvicidal activity of oil-resin fractions from the Brazilian medicinal plant Copaifera reticulata Ducke (Leguminosae-Caesalpinoideae) against Aedes aegypti (Diptera, Culicidae). Revista da Sociedade Brasileira de Medicina Tropical, v.40, n.3, p.264-67, 2007.

Rev. Bras. PI. Med., Campinas, v.15, n.3, p.363-367, 2013. 
SILVA, I.G. et al. Efeito larvicida e toxicológico do extrato bruto etanólico da casca do caule de Magonia pubescens sobre o Aedes aegypti em criadouros artificiais. Revista de Patologia Tropical, v.32, n.1, p.73-86, 2003.

SIMAS, N.K. et al. Produtos naturais para o controle da transmissão da dengue - atividade larvicida de Myrozylon balsamum (óleo vermelho) e de terpenóides e fenilpropanóides. Química Nova, v.27 n.1, p.46-9, 2004.

TAUIL, P.L. Urbanização e ecologia do dengue. Caderno de Saúde Pública, v.17, n.suppl, p.99-102, 2001.
TRIPATIIY, A. et al. The mosquitocidal activity of methanolic extract os Lantana cramera Root and Anacardium occidentale Leaf: role glutathione s-transferase in insecticide resistance. Journal Medical Entomology, v.48, n.2, p.291-295, 2011.

WHO-WORLD HEALTH ORGANIZATION. Dengue guidelines treatment, prevention and control. New ed. Geneva: World Health Organization, 2009. 147p.

ZUCKER, E. Standard evaluation procedure acute toxicity test for freshwater fish. Washington: USEPA, 1985. 17p.

Rev. Bras. PI. Med., Campinas, v.15, n.3, p.363-367, 2013. 